\title{
Identification of key genes and pathways between type I and type II endometrial cancer using bioinformatics analysis
}

\author{
KAI ZHANG ${ }^{*}$, HUIYANG LI ${ }^{*}$, YE YAN, YUQIN ZANG, KE LI, YINGMEI WANG and FENGXIA XUE \\ Department of Gynecology and Obstetrics, Tianjin Medical University General Hospital, Tianjin 300052, P.R. China
}

Received October 29, 2018; Accepted May 17, 2019

DOI: $10.3892 / \mathrm{ol} .2019 .10550$

\begin{abstract}
Endometrial carcinoma (EC) is a common malignant neoplasm of the female reproductive tract. The malignant degree of type II EC is much greater than that of type I EC, usually presenting with a high recurrence rate and a poor prognosis. Therefore, the present study aimed to examine the principal genes associated with the degree of differentiation in type I and type II EC and reveal their potential mechanisms. Differentially expressed genes (DEGs) were selected from the gene expression profiles derived from The Cancer Genome Atlas. Gene Ontology and Kyoto Encyclopedia of Genes and Genomes (KEGG) pathway enrichment analyses were conducted. In the present study, the KEGG pathway enrichment analysis revealed that 5,962 upregulated DEGs were significantly enriched in the 'p53 signaling pathway' and involved in 'lysine degradation'. In addition, 3,709 downregulated DEGs were enriched in 'pathways in cancer', as well as 'tight junction regulation', the 'cell cycle' and the 'Wnt signaling pathway'. The 13 top hub genes MAPK1, PHLPP1, ESR1, MDM2, CDKN2A, CDKN1A, AURKA, BCL2L1, POLQ, PIK3R3, RHOQ, EIF4E and LATS2 were identified via the protein-protein interaction network. Furthermore, the OncoPrint algorithm from cBioPortal declared that $25 \%$ of EC cases carried genetic alterations. The altered DEGs (MAPK1, MDM2, AURKA,EIF4E and LATS2) may be involved in tumor differentiation and may be valuable diagnostic biomarkers. In conclusion, a number of principal genes were identified in the present study that may be determinants of poorly differentiated type II EC carcinogenesis, which may contribute to future research into potential molecular mechanisms. In addition,
\end{abstract}

Correspondence to: Professor Yingmei Wang or Professor Fengxia Xue, Department of Gynecology and Obstetrics, Tianjin Medical University General Hospital, 154 Anshan Road, Heping, Tianjin 300052, P.R. China

E-mail: wangyingmei1978@126.com

E-mail: fengxiaxue1962@gmail.com

*Contributed equally

Key words: bioinformatics method, endometrial carcinoma, core genes, biomarkers, therapeutic targets, survival these genes may help identify candidate biomarkers and novel therapeutic targets for type II EC.

\section{Introduction}

Endometrial carcinoma (EC) occurs in the endometrium and is one of the most common female gynecological tumors. It was estimated that $>63,230$ new cases of EC and nearly 11,350 deaths from EC occurred in 2018 in the USA (1). EC usually includes two types based on its estrogen dependency (2). Generally, the majority of ECs that occur in postmenopausal women are type I, with an increased dependency on estrogen. The prognosis of type I EC is good, and this type accounts for $70-80 \%$ of ECs (3). By contrast, type II EC usually has a poor prognosis. Surgery is the primary treatment for EC that occurs in postmenopausal women. Despite successful surgical intervention, the recurrence rate within 5 years for type II EC is $10-15 \%$, with poor results and short survival time (4).

Similar to that of other tumors, the process of EC initiation, development and metastasis involves genetic aberrations, and changes in the tumor microenvironment (5). The literature has also reported that the development and occurrence of EC are closely associated with a variety of genes and cellular pathways $(6,7)$. However, there is currently little literature available regarding the treatment of type II EC due to the complex nature of the molecular mechanisms and the unfavorable biological behavior. Thus, it is important to study and understand the cellular mechanisms by which type II EC proliferates or initiates apoptosis when searching for diagnostic tools and measures. The present study aimed to identify the difference between type I and type II EC at the genetic level, and to identify candidate biomarkers or therapeutic targets for type II EC.

In life sciences, high-throughput sequencing analysis of gene expression has become increasingly valued as a promising tool for the early diagnosis and grading of cancer, the prediction of prognosis and the discovery of new anticancer targeted drugs. In the present study, by applying genomic techniques, particularly large-scale genome sequencing analyses, the genomic variation map of human EC was drawn and systematically analyzed to find all the minor mutations of carcinogenic and tumor suppressor genes, which can improve the ability to diagnose, treat and prevent cancer. In the present analysis, 177 samples were selected that contained 20,531 gene expression profiles from The Cancer Genome Atlas (TCGA) database. 
Morpheus (https://software.broadinstitute.org/morpheus/) was utilized to select differentially expressed genes (DEGs). Gene Ontology (GO) (8) and the Kyoto Encyclopedia of Genes and Genomes (KEGG) (9) pathway enrichment analyses were then performed to investigate the biological processes, functions and pathways associated with the selected DEGs. Next, the protein-protein interaction (PPI) network was investigated via Cytoscape v3.6.1(http://www.cytoscape.org/) to identify key genes involved in type I and type II EC. In addition, the overall survival (OS) of these the dominant genes was analyzed using the Kaplan-Meier method with GraphPad Prism software version 6.0 (GraphPad Software, Inc.). Finally, the potential associations between genes were analyzed using a visual image according to TCGA database. New clues were identified to guide treatment, with gene-targeted therapy also expected to be an effective treatment for type II EC.

\section{Materials and methods}

Identification of DEGs from TCGA. The gene expression profiles of EC were collected from the TCGA (10) database through cBioPortal (https://www.cbioportal.org/). The Uterine Corpus Endometrial Carcinoma (TCGA, Provisional) which is updated regularly, was downloaded $(11,12)$; it contained the genetic information and clinical data of 547 patients. In total, 177 EC specimens, which included 119 subtype I (endometrioid endometrial adenocarcinoma) and 58 subtype II (serous endometrial adenocarcinoma) samples and corresponded to complete clinical basic information, were included. Thus, the group of 177 cases was a subset of the 547 cohort. The clinical data of the patients, including the demographic features, International Federation of Gynecology and Obstetrics (FIGO) stage (13), and histological type according to the World Health Organization 2014 classification (14), as well as performance status, were retrospectively analyzed. OS and progression-free survival (PFS) were assessed using Kaplan-Meier analyses for the 119 patients with type I disease and the 58 patients with type II disease. Next, the gene expression profile data of type I and type II patients were downloaded from TCGA database and saved in a file, which was uploaded to the Morpheus online tool, which is a versatile matrix visualization and analysis software. A series of processing selection operations were performed, including 'Annotate Selection', 'Maker Selection' and 'T-test'. Finally, the results were presented in the form of a heat map and downloaded. Differential genes with P-values $<0.05$ were screened by heat maps using Morpheus online tools.

Analysis of the DEGs with GO and KEGG online tools. The Database for Annotation, Visualization and Integrated Discovery (DAVID; https://david.ncifcrf.gov/) is a bioinformatics database that integrates biological data and analysis tools to deliver systematic, comprehensive bio-functional annotation information for large-scale gene or protein lists. KEGG (https://www.genome.jp/) is a database that integrates information on genomic, chemical and systemic functions to reveal the genetic and chemical blueprints of life processes (15). In addition, KEGG has powerful capabilities that use graphics to introduce numerous metabolic pathways and associations between pathways. The DAVID online software analyzed the potential pathways of the core genes with $\mathrm{P}$-values $<0.05$.
PPI network construction and module analysis. The Search Tool for the Retrieval of Interacting Genes/Proteins (STRING; http://string-db.org/) is a tool that studies genetic interactions and can assess PPI (16). Therefore, the STRING database (version 10.5) in Cytoscape (version 3.6.1) (17) was used to identify and map potential associations between the DEGs. The cut-off criteria were a confidence score $\geq 0.4$ and a maximum number of iterations equal to 0 . Modules in the PPI network were extracted from Cytoscape using the MCODE, with the following criteria: Degree cut-off, 2; node score cut-off, 0.2; k-core, 2; and maximum depth, 100. On this basis, only modules with a score $>5$ were selected for further function and pathway enrichment analyses. The DAVID tool aided the analysis of the pathways between genes within these modules. In addition, the potential associations between the 13 core genes were assessed using KEGG and GO analysis. These genes with confidence scores between $>0.4$ and $<5$ maximum number of interactions were assigned to STRING.

Potential molecular mechanisms of the top 13 aberrantly expressed genes in EC. cBioPortal (https://www.cbioportal. org/) is a database with integrated genetic data, including DNA mutations, methylations, gene amplifications, homozygous deletions, protein alterations and phosphorous abundance. The present study contains results from 10 published tumor studies and $>20$ results from TCGA. Similarly, the Uterine Corpus Endometrial Carcinoma (TCGA, Provisional) was selected for further analysis in the cBioPortal database. Therefore, possible genetic alterations of the top 13 aberrantly expressed genes in EC were summarized using the visual cBioPortal v1.11.3 analysis tool, and the clinical value following genetic alterations were further analyzed.

Statistical analysis. All statistical analyses were performed using SPSS software (version 22.0; IBM Corp.). Comparisons of the demographics and general characteristics of the EC sample groups were performed using the $\chi^{2}$ and Fisher's exact tests. A paired Student's t-test was used to analyse quantitative data. The survival rate was estimated using the Kaplan-Meier method and the comparison between the type I and type II groups was calculated using the log-rank test. Adjusted P-value was used to find genuine tendencies towards co-occurring genes with very low error rates using the Benjamini-Hochberg false discovery rate (FDR) correction procedure. $\mathrm{P}<0.05$ was considered to indicate a statistically significant result.

\section{Results}

Identification of the DEGs. The heat maps from the Morpheus online tool were used to compare the data of the type I samples with that of the type II samples, and DEGs were determined using the threshold of $\mathrm{P}<0.05$. In total, 9,671 genes were screened as DEGs from the heat maps. Among them, 5,962 genes were identified as being upregulated, whereas 3,709 were downregulated in type I samples when compared with type II samples. The expression heat maps revealing the top 200 genes in the matched 9,671 DEGs are presented in Fig. 1, and detailed information is also presented in Table SI. 

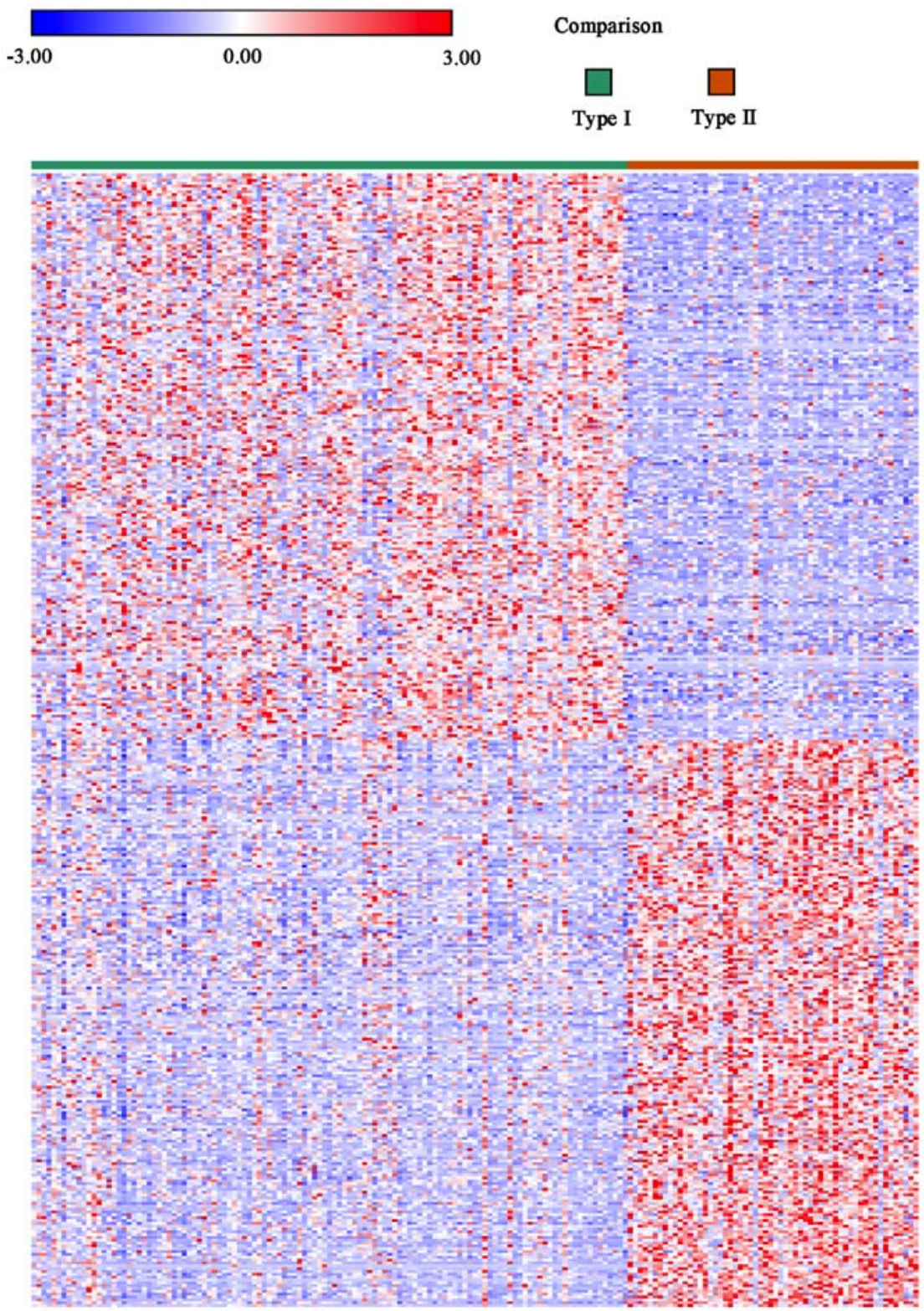

Figure 1. Heat map of the top 200 differentially expressed genes. Red indicates upregulated genes and blue indicates downregulated genes. The top 200 upregulated DEGs and the top 200 downregulated DEGs in type I EC compared with type II EC are shown. DEGS, differentially expressed genes; $\mathrm{EC}$, endometrial carcinoma.

Demographics and clinical characteristics of patients with type I and type II EC. The patients were subdivided into type I and type II groups according to their histopathological type following surgery. In the present study, 119 cases of pathologically confirmed endometrioid adenocarcinoma were selected as the type I group and 58 cases of serous carcinomas were selected as the type II group. There were significant differences in menopausal stage, histological subtype, FIGO stage and vital status. However, there were no significant differences in age, body mass index or ethnicity between the aforementioned two groups. The demographics and clinical characteristics of these two groups are summarized in Table I. The Kaplan-Meier analysis curves of OS of the two groups are presented in Fig. 2A. A significant statistical difference was observed in the OS between the two groups $(\mathrm{P}=0.022)$, with the type I group experiencing increased survival times, whereas no significant difference was revealed in the
PFS time between the two groups, as presented in Fig. 2B $(\mathrm{P}=0.192)$.

GO function and KEGG pathway enrichment analyses. All DEGs were uploaded to the functional annotation tool DAVID Bioinformatics Resources (version 6.7), to identify the over-represented GO categories and KEGG pathways. From the GO analysis, the top 5 biological process (BP), cell component and molecular function results are presented in Table II according to their $\mathrm{P}$-values in reverse order.

The results of the GO analysis revealed that the upregulated DEGs were primarily enriched in the BP for 'positive regulation of apoptosis', 'positive regulation of programmed cell death' and 'positive regulation of cell death', as well as 'induction of apoptosis by intracellular signals' and 'fatty acid metabolic process'. The downregulated DEGs focused on 'transcription, DNA-dependent pathways', 'RNA biosynthetic 
Table I. Comparison of the demographics and general characteristics of endometrial carcinoma sample groups.

\begin{tabular}{lccc}
\hline Characteristics & Type I & Type II & P-value \\
\hline Total patients, n & 119 & 58 & \\
Age range, years & $33-88$ & $51-90$ & 0.068 \\
BMI & $35.29 \pm 19.48$ & $30.02 \pm 7.90$ & 0.065 \\
Menopausal, n (\%) & & & 0.019 \\
Pre- & $14(11.76)$ & $0(0.00)$ & \\
Post- & $96(80.67)$ & $51(87.93)$ & \\
Data not available & $9(7.56)$ & $7(12.07)$ & \\
Ethnicity, n (\%) & & & 0.466 \\
Caucasian & $58(48.74)$ & $34(58.62)$ & \\
African-American & $43(36.13)$ & $17(29.31)$ & \\
Other & $18(15.13)$ & $7(12.07)$ & \\
Histological subtype, n (\%) & & & 0.001 \\
Grade 1 & $13(10.92)$ & $1(1.72)$ & \\
Grade 2 & $19(15.97)$ & $1(1.72)$ & \\
Grade 3 & $87(73.11)$ & $56(96.55)$ & \\
FIGO stage, n (\%) & & & $<0.001$ \\
I & $75(63.03)$ & $24(41.38)$ & \\
II & $19(15.97)$ & $4(6.90)$ & \\
III & $22(18.49)$ & $23(39.66)$ & \\
IV & $3(2.52)$ & $7(12.07)$ & \\
Vital status, n (\%) & & & 0.022 \\
Alive & $102(85.71)$ & $41(70.69)$ & \\
Dead & $17(14.29)$ & $17(29.31)$ & \\
\hline
\end{tabular}

Including Asian, Native Hawaiian or Pacific Islander and American-Indian or Native Alaskan. FIGO, International Federation of Gynecology and Obstetrics; BMI, body mass index.

process', 'apoptosis', 'programmed cell death' and 'apoptotic mitochondrial changes'. It was revealed that the upregulated DEGs were significantly enriched in the "p53 signaling pathway' and 'lysine degradation' processes, while the downregulated DEGs were enriched in 'pathways in cancer', 'tight junction' regulation, the 'cell cycle', the 'Wnt signaling pathway', 'chronic myeloid leukemia' development and 'small-cell lung cancer' development according to the results of the KEGG pathway enrichment analysis (Table III).

Screening for core genes and modules via the PPI network. An MCODE plug-in was used in Cytoscape to build a PPI network with 212 nodes and 380 edges (Fig. 3A). Using the Network Analyzer tool, the top 13 hub DEGs with the highest node degrees were obtained (Table IV). As presented in Table IV, the hub genes were MAPK1, PHLPP1, ESR1, MDM2, CDKN2A, CDKN1A, AURKA, BCL2L1, POLQ, PIK3R3, RHOQ, EIF4E and LATS2. A PPI network of the top 13 central genes with the highest connectivity was established through the STRING protein analysis (Fig. 3B).

In addition, the MCODE plug-in extracted an important module with a score $>4$, and then performed function and pathway enrichment analyses (Fig. 4A). This module consisted of 10 nodes and 41 edges, which were primarily involved in the 'positive regulation of apoptosis', 'positive regulation of programmed cell death' and 'positive regulation of cell death' (Table V). Three other modules were also revealed (Fig. 4B-D), whose further function and pathway enrichment analysis data were not available in the GO and KEGG databases.

Top 13 expression genes in EC. The OncoPrint module in cBioPortal, an online tool, was used to analyze genetic alterations, and showed that 25\% (136/547) of the cases had undergone genetic changes (Fig. 5A). The types of genetic alterations included amplification, deep deletion and mRNA upregulation. Notably, patients with genetic alterations had shorter OS and PFS times than those without alterations (log rank, $\mathrm{P}=0.0179$; Fig. 5B; and log rank, $\mathrm{P}=0.0379$; Fig. 5C, respectively). Furthermore, as presented in Table VI, there were 6 pairs of genes identified via cBioPortal with significant tendencies towards co-occurrence. These genes were PHLPP1 and LATS2, POLQ and LATS2, PHLPP1 and POLQ, ESR1 and CDKN1A, PHLPP1 and PIK3R3, and POLQ and ESR1.

\section{Discussion}

$\mathrm{EC}$ is a common malignant neoplasm of the female reproductive tract (1). The histological type of disease, determined postoperatively, has a crucial impact on the survival and prognosis of patients with EC. Pathologically determined serous carcinoma usually leads to a poor prognosis with metastasis and more difficult surgery. Furthermore, the 5-year survival rate only reaches $25-30 \%$ (18). Consequently, a comprehensive understanding of the molecular mechanism behind the occurrence and development of $\mathrm{EC}$ is of importance for management and therapy, as is the development of novel targeted therapies to improve OS time. With the widespread use of next-generation sequencing technologies and microarrays, the expression levels of genes in the human genome are openly included in public databases. Therefore, the molecular mechanisms of different pathological types of EC and microarray data can be investigated in order to facilitate the genetic research of EC.

In the present study, it was observed through GO analysis that upregulated DEGs were primarily enriched in the 'positive regulation of apoptosis', 'positive regulation of programmed cell death', 'positive regulation of cell death', 'induction of apoptosis by intracellular signals' and 'fatty acid metabolic process', and that the downregulated DEGs were primarily involved in 'transcription, DNA-dependent pathways', 'RNA biosynthetic process', 'apoptosis', 'programmed cell death' and 'apoptotic mitochondrial changes'. These results are compatible with the suggestion that the main causes of tumor development and metastasis are defective functions in the regulation of apoptosis and cell death regulators (19). The metabolic patterns, such as that of glycolysis, oxidative phosphorylation, amino acid metabolism, fatty acid metabolism and nucleic acid metabolism, are significantly altered during cell carcinogenesis. Among these processes, fatty acid metabolism is essential for energy storage, membrane proliferation and the synthesis of signaling molecules (20). Thus, studying the mechanism of de novo fatty acid synthesis and its association with the development and progression of tumors 

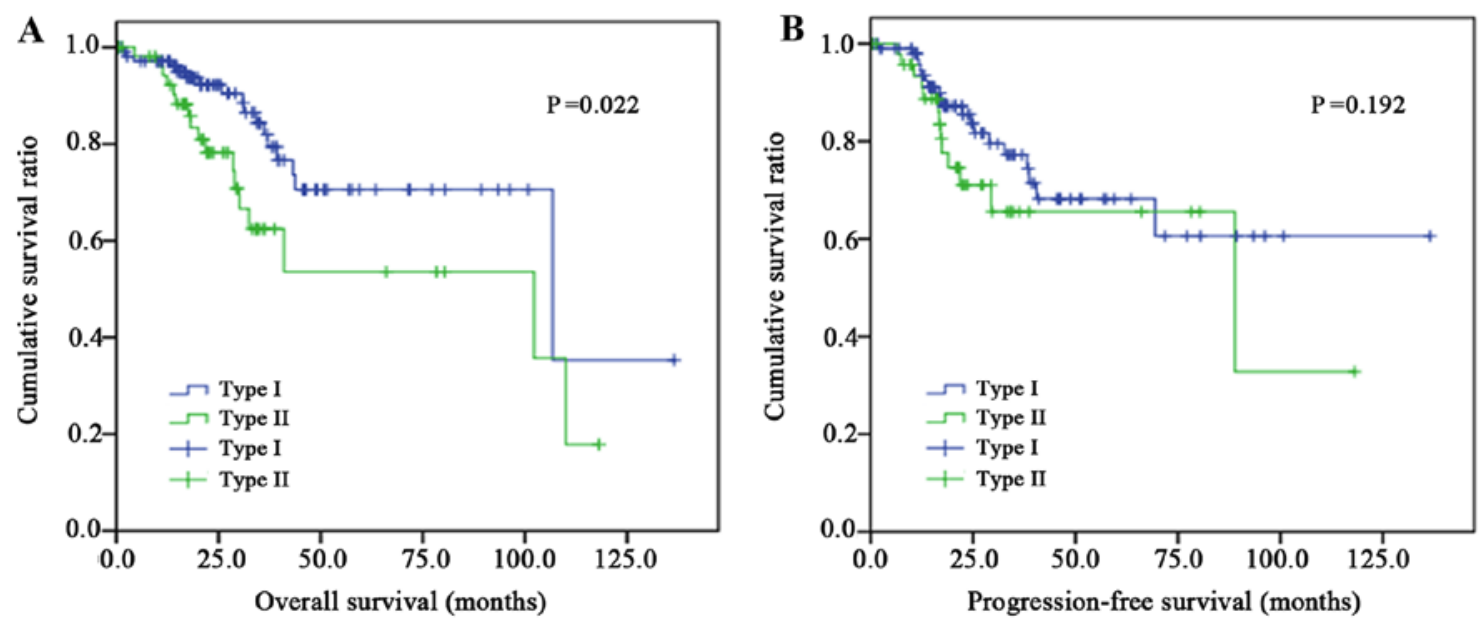

Figure 2. Comparison of overall survival and progression-free survival in patients with type I and type II endometrial cancer. (A) Kaplan-Meier analysis curves of overall survival of 119 patients in the type I group versus 58 patients in the type II group. Significant statistical difference was noted in the overall survival times between the two groups ( $\mathrm{P}=0.022)$. (B) Kaplan-Meier analysis curves of progression-free survival of 119 patients in the type I group versus 58 in the type II group. There was also no statistical difference identified in the progression-free survival times between these two groups $(\mathrm{P}=0.192)$.

may be a new idea for improving tumor diagnosis, prevention and treatment. A previous study revealed that the risk of EC is significantly reduced by the consumption of $\omega-3$ polyunsaturated fatty acids (PUFAs) in high-fat diets. However, the underlying molecular mechanism of action of PUFAs in EC is not well understood (21). Furthermore, the latest research has also demonstrated that $\omega-6$ is strongly associated with the risk of breast cancer $(22,23)$.

In addition, upregulated DEGs are primarily involved in the 'p53 signaling pathway' and the 'lysine degradation' processes in the KEGG pathway enrichment analysis. Previous studies have revealed that markers of the p53 pathway improved the stratification of EC and can provide novel insights into the effect of this pathway in the morphological classification of high-risk EC $(24,25)$. Furthermore, the phenomenon that the p53 signaling pathway is disturbed in EC has been widely noted. In addition, lysine-specific demethylase 1 (LSD1) plays a vital role in the regulation of chromatin and can affect the occurrence and development of many types of malignant tumor by regulating the proliferation, invasion and metastasis of tumor cells. Therefore, the special role of LSD1 allows for it to become a new antitumor target (26). The downregulated DEGs were enriched in 'pathways in cancer', 'tight junction' regulation, the 'cell cycle', the 'Wnt signaling pathway', 'chronic myeloid leukemia' development' and 'small-cell lung cancer' development. As reported in the literature, alteration of pathways such as the Janus kinase/signal transducer and activator of transcription proteins signaling pathway, the Wnt signaling pathway and the phosphoinositide 3-kinase (PI3K)/protein kinase $\mathrm{B}(\mathrm{Akt}) / \mathrm{mammalian}$ target of rapamycin pathway were verified in a number of different types of cancer $(27,28)$. For example, stabilizing DVL3 expression can activate the Wnt/ $\beta$-catenin signaling pathway in hepatocellular carcinoma (29), and E2F3 expression, as a potent transcriptional inducer of cell-cycle progression, can promote non-small cell lung cancer progression through the microRNA-377-3p-E2F3 pathway (30). These altered DEGs may be co-expressed in different types of cancer and participate in tumorigenesis, such as that of chronic myeloid leukemia and small cell lung cancer, according to KEGG pathway analysis. In the multi-stage development of cancer, the imbalance of the equilibrium steady state of the activity of signaling pathways responsible for cell cycle regulation and division will lead to the inhibition of apoptosis and the enhancement of cell proliferation (31). Tight junctions are protein structures that control the transport of water, ions and macromolecules across cell layers (32). Previous studies have demonstrated that low levels of tight junction plaque molecules such as zonula occludens-1 and multi-PDZ domain protein-1 in breast cancer are associated with a poor prognosis $(33,34)$. The Wnt signaling pathway is a highly conserved and complicated network, in which the abnormal activation of the canonical Wnt/ $\beta$-catenin pathway can lead to the anomalous expression of tumor-associated genes and impact EC progression. Chen et al (35) reported that $\beta$-catenin and c-myc were activated due to the upregulation of Wnt10b expression, which ultimately promoted the proliferation of Ishikawa cells and inhibited cell apoptosis. Inhibiting the canonical Wnt/ $\beta$-catenin pathway or interfering with the regulation of its upstream signals may be a target for anticancer therapy (36-38).

According to the results of the PPI network with DEGs in the present study, the 13 top hub genes are as follows: MAPK1, PHLPP1, ESR1, MDM2, CDKN2A, CDKN1A, AURKA, BCL2L1, POLQ, PIK3R3, RHOQ, EIF4E and LATS2.

The MAPK1 gene, which encodes a member of the MAPK family, is involved in cell proliferation, differentiation and apoptosis. In addition, MAPKs are also known to be extracellular signal-regulated kinases that act as an integration point for biochemical signals. MAPK1 is overexpressed in various solid tumors, such as EC, non-small cell lung cancer, prostate cancer and colorectal cancer $(39,40)$. Wang et al (41) demonstrated that insulin receptors are activated by visfatin, which in turn activates the PI3K/Akt and MAPK/ERK signaling pathways. This process is thought to be one of the molecular mechanisms by which visfatin promotes the progression of EC.

PHLPP1 is encoded by a member of the serine/threonine phosphatase family. The function of encoded proteins is to act 
Table II. GO analysis of differentially expressed genes associated with patients with different types of endometrial carcinoma.

\begin{tabular}{|c|c|c|c|c|}
\hline Category & Term & Count & $\%$ & P-value \\
\hline \multicolumn{5}{|l|}{ Upregulated } \\
\hline GOTERM_BP_FAT & GO:0043065 positive regulation of apoptosis & 12 & 0.8 & $6.9 \times 10^{-4}$ \\
\hline GOTERM_BP_FAT & GO:0043068 positive regulation of programmed cell death & 12 & 0.8 & $7.3 \times 10^{-4}$ \\
\hline GOTERM_BP_FAT & GO:0010942 positive regulation of cell death & 12 & 0.8 & $7.6 \times 10^{-4}$ \\
\hline GOTERM_BP_FAT & GO:0008629 induction of apoptosis by intracellular signals & 5 & 0.3 & $9.3 \times 10^{-4}$ \\
\hline GOTERM_BP_FAT & GO:0006631 fatty acid metabolic process & 8 & 0.5 & $1.1 \times 10^{-3}$ \\
\hline GOTERM_CC_FAT & GO:0009898 internal side of plasma membrane & 7 & 0.4 & $4.1 \times 10^{-2}$ \\
\hline GOTERM_MF_FAT & GO:0017137 Rab GTPase binding & 3 & 0.2 & $2.5 \times 10^{-2}$ \\
\hline GOTERM_MF_FAT & GO:0008565 protein transporter activity & 4 & 0.3 & $3.4 \times 10^{-2}$ \\
\hline GOTERM_MF_FAT & $\begin{array}{l}\text { GO:0003705 RNA polymerase II transcription factor activity, } \\
\text { enhancer binding }\end{array}$ & 3 & 0.2 & $4.0 \times 10^{-2}$ \\
\hline GOTERM_MF_FAT & GO:0019207 kinase regulator activity & 4 & 0.3 & $4.0 \times 10^{-2}$ \\
\hline GOTERM_MF_FAT & GO:0003857 3-hydroxyacyl-CoA dehydrogenase activity & 2 & 0.1 & $4.8 \times 10^{-2}$ \\
\hline \multicolumn{5}{|l|}{ Downregulated } \\
\hline GOTERM_BP_FAT & GO:0006351 transcription, DNA-dependent & 7 & 0.6 & $1.1 \times 10^{-2}$ \\
\hline GOTERM_BP_FAT & GO:0032774 RNA biosynthetic process & 7 & 0.6 & $1.2 \times 10^{-2}$ \\
\hline GOTERM_BP_FAT & GO:0006915 apoptosis & 10 & 0.8 & $1.5 \times 10^{-2}$ \\
\hline GOTERM_BP_FAT & GO:0012501 programmed cell death & 10 & 0.8 & $1.6 \times 10^{-2}$ \\
\hline GOTERM_BP_FAT & GO:0008637 apoptotic mitochondrial changes & 3 & 0.2 & $1.7 \times 10^{-2}$ \\
\hline GOTERM_CC_FAT & GO:0005654 nucleoplasm & 16 & 1.3 & $4.9 \times 10^{-4}$ \\
\hline GOTERM_CC_FAT & GO:0070013 intracellular organelle lumen & 24 & 1.9 & $7.2 \times 10^{-4}$ \\
\hline GOTERM_CC_FAT & GO:0031981 nuclear lumen & 21 & 1.7 & $7.9 \times 10^{-4}$ \\
\hline GOTERM_CC_FAT & GO:0044451 nucleoplasm part & 12 & 1.0 & $8.9 \times 10^{-4}$ \\
\hline GOTERM_CC_FAT & GO:0043233 organelle lumen & 24 & 1.9 & $9.9 \times 10^{-4}$ \\
\hline GOTERM_MF_FAT & GO:0000166 nucleotide binding & 27 & 2.2 & $2.2 \times 10^{-3}$ \\
\hline GOTERM_MF_FAT & GO:0005524 ATP binding & 19 & 1.5 & $7.3 \times 10^{-3}$ \\
\hline GOTERM_MF_FAT & GO:0032559 adenyl ribonucleotide binding & 19 & 1.5 & $8.4 \times 10^{-3}$ \\
\hline GOTERM_MF_FAT & GO:0030554 adenyl nucleotide binding & 19 & 1.5 & $1.4 \times 10^{-2}$ \\
\hline GOTERM_MF_FAT & GO:0032555 purine ribonucleotide binding & 21 & 1.7 & $1.5 \times 10^{-2}$ \\
\hline
\end{tabular}

GO, Gene Ontology; BP, biological processes; CC, cell component; MF, molecular function.

Table III. Kyoto Encyclopedia of Genes and Genomes pathway analysis of differentially expressed genes associated with patients with different types of endometrial carcinoma.

\begin{tabular}{|c|c|c|c|c|}
\hline Term & Count & $\%$ & P-value & Genes \\
\hline \multicolumn{5}{|l|}{ Upregulated } \\
\hline hsa04115: p53 signaling pathway & 5 & 0.3 & $5.6 \times 10^{-3}$ & CDKN1A, TNFRSF10B, BBC3, SESN1, TP73 \\
\hline hsa00310: Lysine degradation & 3 & 0.2 & $3.4 \times 10^{-2}$ & AADAT, DOT1L, HADH \\
\hline \multicolumn{5}{|l|}{ Downregulated } \\
\hline hsa05200: Pathways in cancer & 10 & 0.8 & $2.7 \times 10^{-4}$ & $\begin{array}{l}\text { DVL3, ACVR1B, E2F3, CDKN2A, CDKN2B, } \\
\text { LAMA5, PAX8, TFG, BCL2L1, WNT7A }\end{array}$ \\
\hline hsa04530: Tight junction & 4 & 0.3 & $7.8 \times 10^{-3}$ & PARD6B, CLDN6, PRKCI, TJAP1 \\
\hline hsa04110: Cell cycle & 5 & 0.4 & $1.0 \times 10^{-2}$ & E2F3, CDKN2A, CDKN2B, TFDP2, TTK \\
\hline hsa04310: Wnt signaling pathway & 4 & 0.3 & $1.1 \times 10^{-2}$ & SENP2, DVL3, TBL1XR1, WNT7A \\
\hline hsa05220: Chronic myeloid leukemia & 4 & 0.3 & $1.4 \times 10^{-2}$ & ACVR1B, E2F3, CDKN2A, BCL2L1 \\
\hline hsa05222: Small cell lung cancer & 4 & 0.3 & $2.3 \times 10^{-2}$ & E2F3, CDKN2B, LAMA5, BCL2L1 \\
\hline
\end{tabular}

as tumor inhibitors in a number of different types of cancer, and their possible molecular mechanism is to regulate apoptosis by dephosphorylation and inactivate the serine/threonine kinase Akt. Thus, PHLPP1 has an effect on mediating the activation 


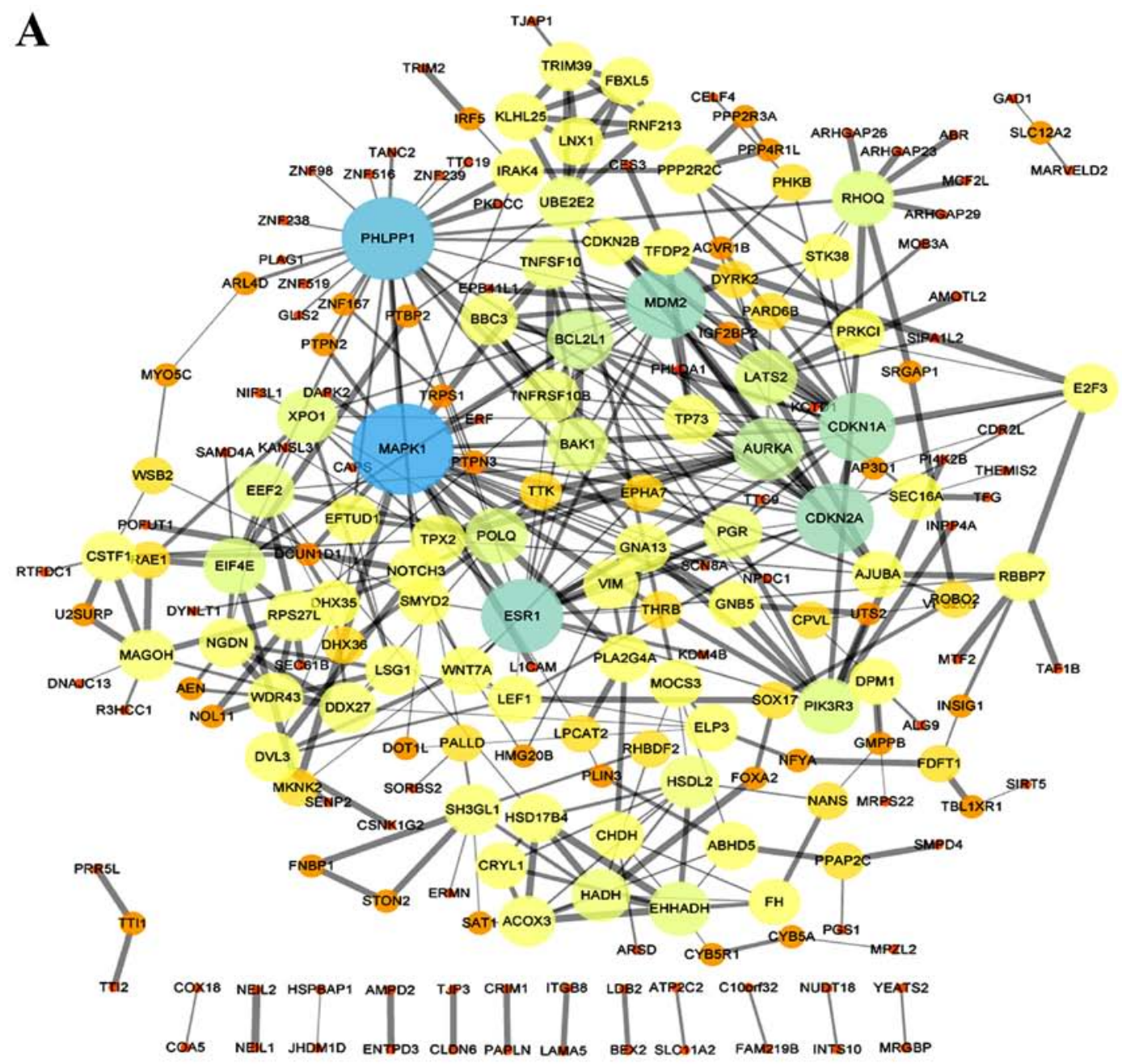

B

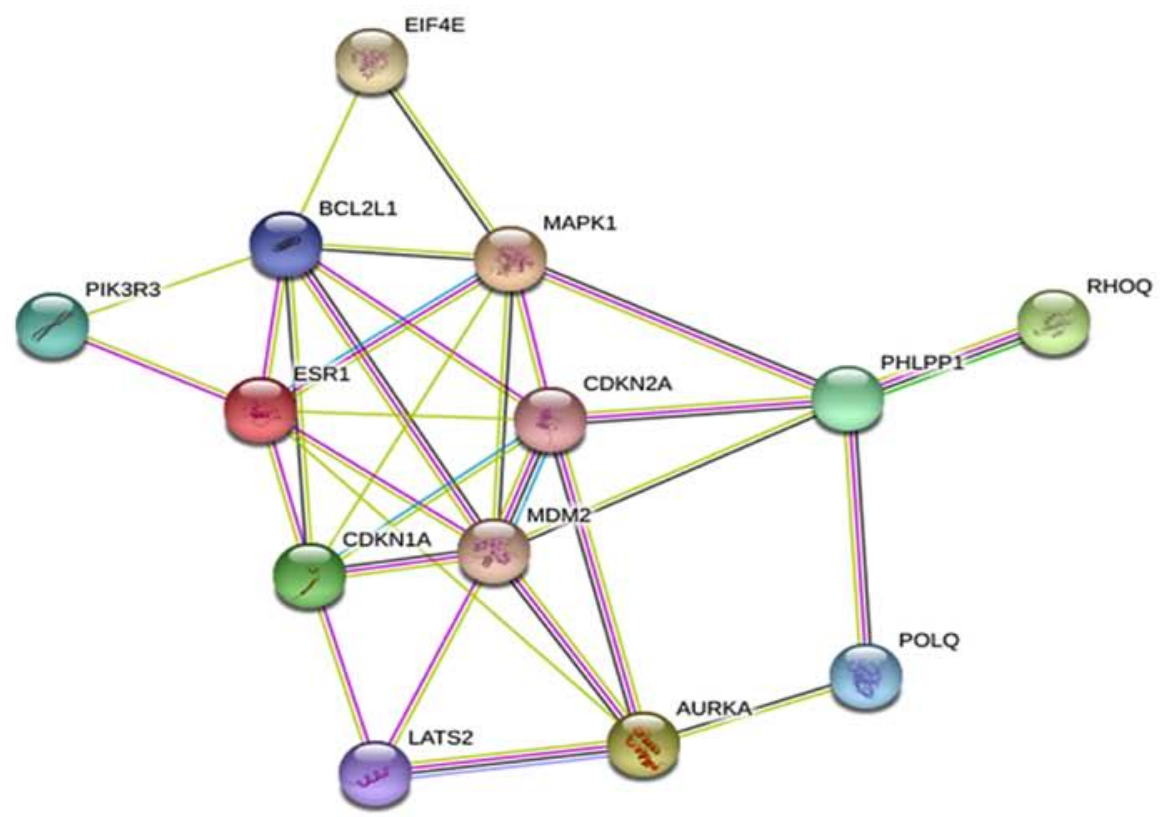

Figure 3. PPI network of the differentially expressed genes and the top 13 hub genes. (A) PPI network and hub genes of the differentially expressed genes. Nodes were given different colors according to the interaction values between two genes. Yellow nodes represent a low value (darker node represents a smaller value) whereas blue nodes represent a high value (darker node represents a higher value). (B) PPI network of the top 13 hub genes. PPI, protein-protein interaction.

of the PI3K/AKT signaling pathway (42). It has been reported that significant downregulation or loss of PHLPP1 is closely associated with metastasis in a variety of different types of tumor, such as colorectal cancer, breast cancer and gastric cancer $(43,44)$. This finding coincides with the results from the present study, in that PHLPP1 was more significantly 
Table IV. Top 13 hub genes of the protein-protein interaction network with higher degrees of connectivity.

\begin{tabular}{|c|c|c|}
\hline Hub gene & Gene name & Node degree \\
\hline MAPK1 & Mitogen-activated protein kinase 1 & 32 \\
\hline PHLPP1 & $\mathrm{Ph}$ domain and leucine-rich repeat protein phosphatase 1 & 27 \\
\hline ESR1 & Estrogen receptor 1 & 21 \\
\hline MDM2 & Mdm2 proto-oncogene & 20 \\
\hline CDKN2A & Cyclin-dependent kinase inhibitor 2a (Melanoma, p16, Inhibits Cdk4) & 19 \\
\hline CDKN1A & Cyclin-dependent kinase inhibitor 1a & 18 \\
\hline AURKA & Aurora kinase a & 14 \\
\hline BCL2L1 & Bcl-2-like 1 & 12 \\
\hline POLQ & Dna polymerase $\tau$ & 10 \\
\hline PIK3R3 & Phosphoinositide-3-kinase regulatory subunit 3 & 10 \\
\hline RHOQ & Ras homolog family member $\mathrm{q}$ & 10 \\
\hline EIF4E & Eukaryotic translation initiation factor $4 \mathrm{e}$ & 10 \\
\hline LATS2 & Large tumor suppressor kinase 2 & 10 \\
\hline
\end{tabular}
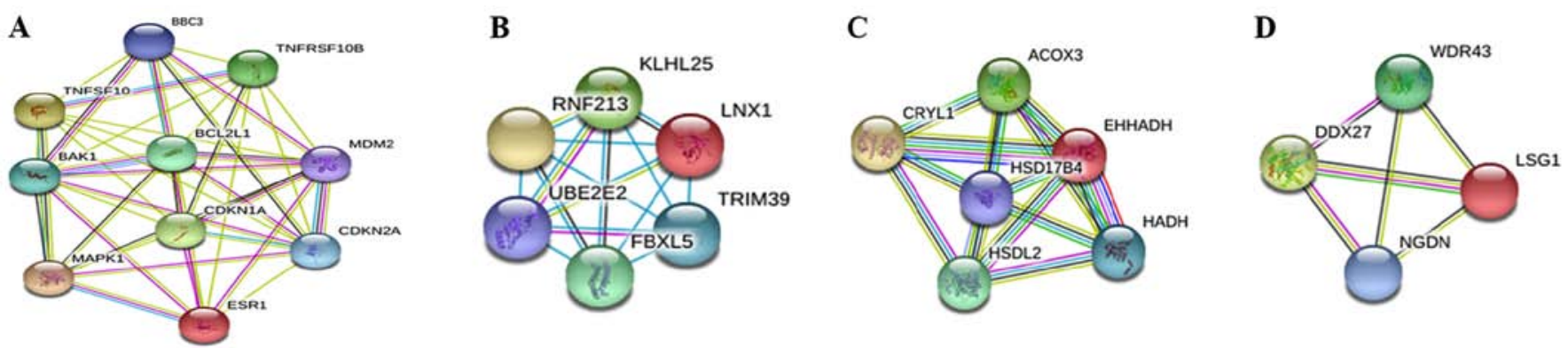

Figure 4. Significant module and enrichment analyses from the protein-protein interaction network. Only modules with a score $>4$ were presented. (A) Module 1. (B) Module 2. (C) Module 3. (D) Module 4.

downregulated in type II EC than in type I EC. PHLPP1 phosphatases have a clinically relevant role in the pathogenesis of insulin resistance-associated diseases, including EC (45). To the best of our knowledge, there has been no study to date regarding the role of PHLPP1 in EC.

The third hub gene, ESR1, encodes an estrogen receptor and has been noted in several pathological processes of breast cancer and endometrial cancer (46). ESR1 is an estrogen-dependent transcription factor discovered by Jensen in 1971 (47). ESR1 is also a member of the nuclear receptor superfamily, which can bind to estrogen, transfer to the nucleus of breast cancer cells and participate in the regulation of gene transcription signaling pathways, thus promoting the proliferation of breast cancer cells (48). The ESR1 gene has been a focus of breast cancer studies for quite some time, but it is also clinically relevant in endometrial, ovarian and other types of cancer.

The MDM2 gene is an oncogene closely associated with the function of p53. MDM2 has been studied in regard to tumors such as lung cancer and uterine sarcoma (49), but its mechanism in the occurrence and development of EC has rarely been reported. The MDM2 protein is overexpressed in both primary and metastatic EC, which also indicates that MDM2 protein expression is closely associated with the development of EC (50). In addition, Wu and Luo (51) revealed that the MDM2 rs2279744 polymorphism was significantly associated with EC risk in the Chinese Han population.

CDKN1A is a gene that encodes a potent inhibitor of cyclin. The encoded protein CDKN1A regulates the progression of the cell cycle and focuses on the $G_{1}$ phase of cell division, as it directly and indirectly binds to and inhibits the activity of complexes with cyclin-dependent kinase 2 or cyclin-dependent kinase 4 (52). The diseases associated with CDKN1A include bladder cancer (53) and pancreatic inflammation (54). The loss of CDKN2A has been demonstrated to be an important event in various types of tumor. Although no clinical trials have been conducted on targeted therapies as of yet, the impact of CDKN1A on the prognosis and chemotherapy resistance in pancreatic and breast cancer have been studied (55).

A protein that seems to mediate microtubule formation and/or stability at the spindle end during chromosome segregation is encoded by the AURKA gene, which is essentially a protein kinase capable of regulating the self-reporting cycle. Recent evidence indicates that the overexpression of AURKA is associated with high tumor grade, severe histological type and paclitaxel sensitivity in EC (56). Furthermore, previous studies have demonstrated that patients with EC who are resistant to conventional surgical treatment and chemotherapy have short survival 
Table V. GO and Kyoto Encyclopedia of Genes and Genomes enrichment analyses of genes in the significant module, with only the top 3 BPs presented.

\begin{tabular}{|c|c|c|c|}
\hline Category & Term & P-value & Genes \\
\hline GOTERM_BP_FAT & $\begin{array}{l}\text { GO:0043065 positive regulation } \\
\text { of apoptosis }\end{array}$ & $1.1 \times 10^{-9}$ & $\begin{array}{l}\text { BAK1, MAPK1, CDKN1A, TNFSF10, CDKN2A, } \\
\text { TNFRSF10B, BBC3, BCL2L1 }\end{array}$ \\
\hline GOTERM_BP_FAT & $\begin{array}{l}\text { GO:0043068 positive regulation } \\
\text { of programmed cell death }\end{array}$ & $1.1 \times 10^{-9}$ & $\begin{array}{l}\text { BAK1, MAPK1, CDKN1A, TNFSF10, CDKN2A, } \\
\text { TNFRSF10B, BBC3, BCL2L1 }\end{array}$ \\
\hline GOTERM_BP_FAT & $\begin{array}{l}\text { GO:0010942 positive regulation } \\
\text { of cell death }\end{array}$ & $1.2 \times 10^{-9}$ & $\begin{array}{l}\text { BAK1, MAPK1, CDKN1A, TNFSF10, CDKN2A, } \\
\text { TNFRSF10B, BBC3, BCL2L1 }\end{array}$ \\
\hline KEGG_PATHWAY & hsa04115: p53 signaling pathway & $9.9 \times 10^{-7}$ & CDKN1A, CDKN2A, TNFRSF10B, BBC3, MDM2 \\
\hline KEGG_PATHWAY & hsa05220: Chronic myeloid leukemia & $1.5 \times 10^{-6}$ & MAPK1, CDKN1A, CDKN2A, MDM2, BCL2L1 \\
\hline KEGG_PATHWAY & hsa05219: Bladder cancer & $1.8 \times 10^{-5}$ & MAPK1, CDKN1A, CDKN2A, MDM2, \\
\hline
\end{tabular}

GO, Gene Ontology; BP, biological process.

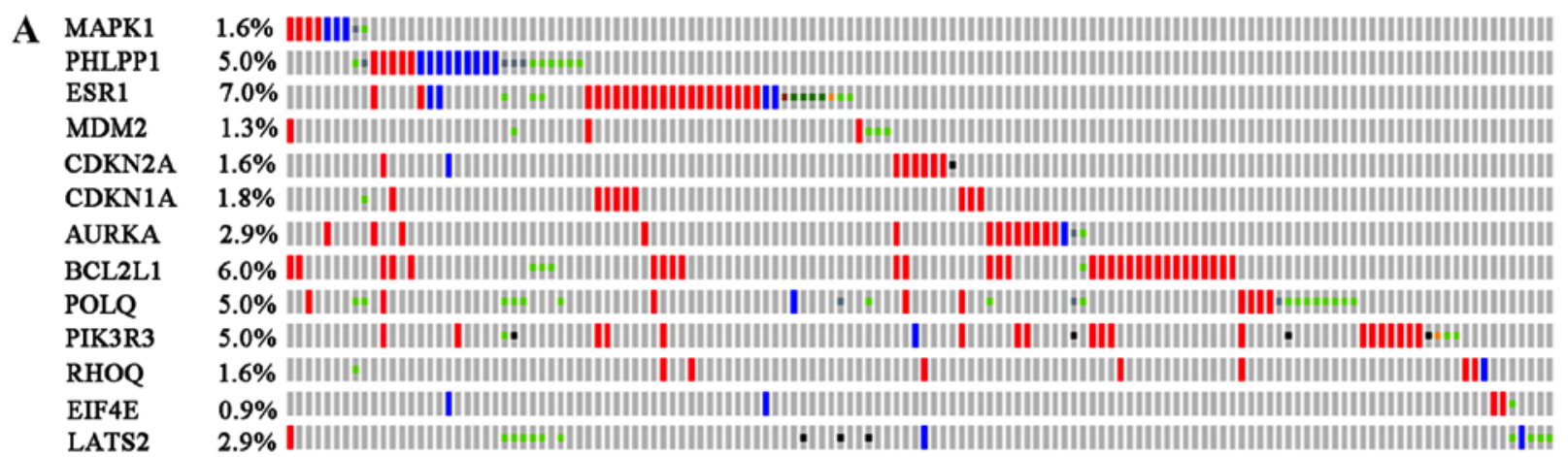

Genetic alteration =Inframe mutation (putative driver)-Inframe mutation (unknown significance)= Missense mutation -Missense mutation (unknown significance)-Truncation mutation = Truncation mutation (unknown significance) | Amplication | Deep deletion || No alterations
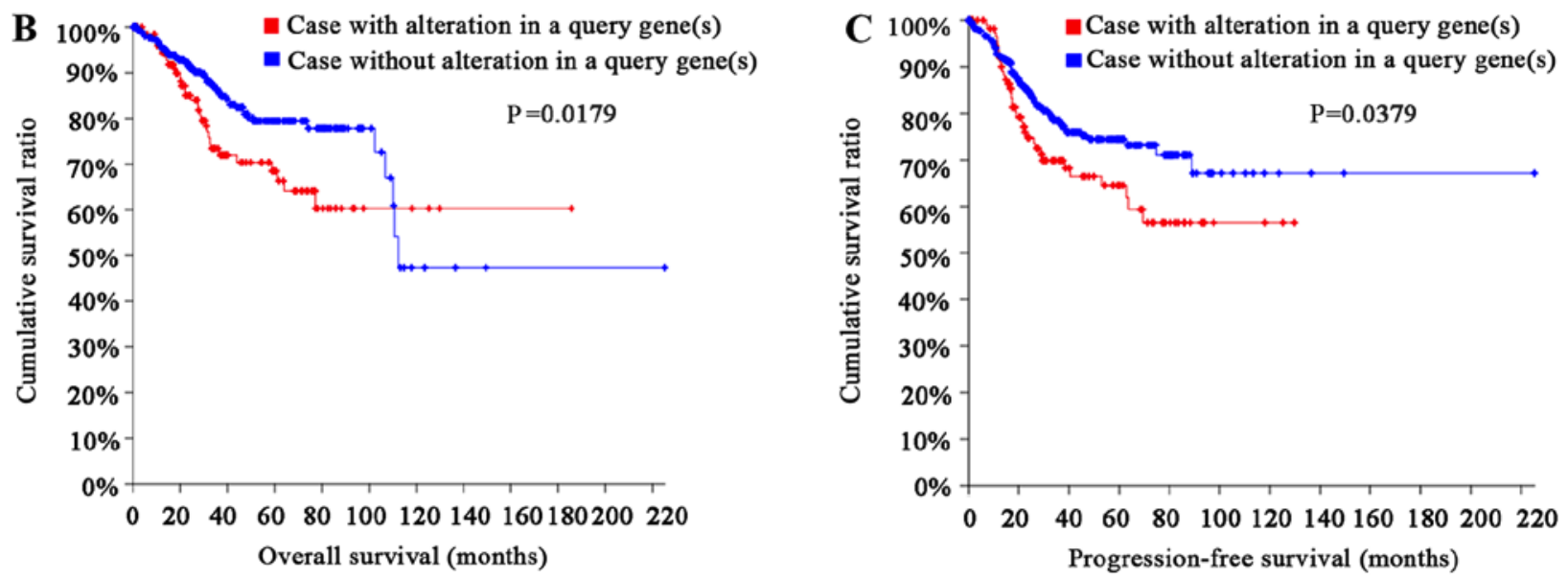

Figure 5. (A) Genetic alterations. Red represents amplification and blue represents deep deletion of genes in 136 of the 547 endometrial carcinoma patients (25\%). The aberrant expression threshold was defined as z-score \pm 2.0 from The Cancer Genome Atlas RNA Sequencing version 2 data. A Kaplan-Meier curve was created between groups with and without alterations. (B) Overall survival Kaplan-Meier estimate. (C) Progression-free survival Kaplan-Meier estimate. The red and blue lines represent cases with and without alterations, respectively.

times and poor prognosis (57). However, the combination of an AURKA inhibitor and paclitaxel can be effective in these patients with EC, so it is speculated that AURKA may be a promising target in EC therapy (58).
Another hub gene, BCL2L1 is a member of the BCL-2 protein family and forms hetero- or homodimers to act as anti-apoptotic or pro-apoptotic regulators that are involved in programmed cell death or apoptosis activities (59). Studies 
Table VI. Results of mutual exclusivity and co-occurrence analysis by cBioPortal.

\begin{tabular}{|c|c|c|c|c|}
\hline Gene A & Gene B & P-value & Adjusted P-value & Association \\
\hline PHLPP1 & LATS2 & $<0.001$ & 0.002 & Tendency towards co-occurrence \\
\hline POLQ & LATS2 & $<0.001$ & 0.007 & Tendency towards co-occurrence \\
\hline PHLPP1 & POLQ & $<0.001$ & 0.014 & Tendency towards co-occurrence \\
\hline ESR1 & CDKN1A & $<0.001$ & 0.014 & Tendency towards co-occurrence \\
\hline PHLPP1 & PIK3R3 & $<0.001$ & 0.030 & Tendency towards co-occurrence \\
\hline POLQ & ESR1 & $<0.001$ & 0.046 & Tendency towards co-occurrence \\
\hline MDM2 & LATS2 & $<0.001$ & 0.052 & Tendency towards co-occurrence \\
\hline AURKA & BCL2L1 & 0.002 & 0.142 & Tendency towards co-occurrence \\
\hline ESR1 & LATS2 & 0.002 & 0.186 & Tendency towards co-occurrence \\
\hline PHLPP1 & BCL2L1 & 0.003 & 0.214 & Tendency towards co-occurrence \\
\hline
\end{tabular}

The analysis contained 22 gene pairs with mutually exclusive alterations (none significant) and 56 gene pairs with co-occurring alterations (18 significant and only the top 10 were shown).

have revealed that both lung cancer and pancreatic cancer are associated with BCL2L1. The associated pathways of BCL2L1 include apoptosis regulation, signal transduction and chronic myeloid leukemia progression (60).

PIK3R3 serves as a second messenger in growth signaling pathways; it encodes the regulatory subunit PI3K that can activate (phosphorylate) tyrosine protein kinases via binding to the $\mathrm{SH} 2$ domain (61). A previous study (62) revealed that the mRNA expression and the expression of PIK3R3 was markedly increased in ovarian cancer samples compared with that in normal ovarian samples, and the difference was statistically significant. Cellular experiments further demonstrated that the proliferation, migration and invasion of the ovarian cancer SKOV3 cells were regulated by the interaction between HOTAIR and PIK3R3 (63). However, given the limited research available, it is not possible to speculate on the association between PIK3R3 and EC.

Furthermore, the small GTPases that are encoded by RHOQ belong to the $\rho$ family. GTPases alternate between inactive GDP and the active GTP binding state, and act as a molecular switch in the signal transduction cascade. The associated pathways include ERK signaling and Akt signaling. In diffuse subtypes of gastric cancer, the overexpression of the RHOQ gene is correlated with a poor prognosis (64).

One of the proteins that make up the eukaryotic translation initiation factor 4F complex is encoded by the EIF4E gene, which can recognize mRNAs through the structure of the 7-methylguanosine cap at the 5' tail. The overexpression of the EIF4E gene and high expression levels of its proteins were noted in the EC specimens, and were associated with FIGO stage, histological grade and degree of lymphatic metastasis (65). The positive expression rates of eIF4E were not only associated with the EC stages as determined by FIGO stage but were also associated with tumor cell differentiation degree and lymphatic metastasis (66). As noted, EIF4E may serve a crucial role in the pathogenesis of EC and become a potential trigger in carcinogenesis.

LATS2 is a tumor suppressor that encodes the corresponding protein kinase, namely, serine/threonine protein kinase. LATS2 mediates the mutation of normal cells into tumor cells by regulating the cell cycle, proliferation and apoptosis of tumor cells. LATS2 not only interacts with p53 in a positive feedback loop to participate in the response to cytoskeletal injury, but LATS2 is also regarded as a co-inhibitory factor with androgen. By limiting proliferation and promoting apoptosis, negative regulators of YAP1 involved in the regulation of the Hippo signaling pathway play a crucial role in organ size control and tumor inhibition (67). Zhao et al (68) showed that prostatic invasion and metastasis were notably downregulated by LATS2 expression in the LAST2-Yap1 signaling pathway. Notably, in a previous study, LATS2 was confirmed as the target of MIR31 in EC cells, as LATS2 was downregulated among MIR31-overexpressing cells (69). These studies were consistent with the results from the present study, showing that LATS2 was significantly downregulated in type II EC.

Currently, the biological functions of CDKN2A, BCL2L1, POLQ and RHOQ in EC are unknown.

Module analysis of the PPI network revealed that the progression and histological types of EC were positively associated with the regulation of apoptosis and programmed cell death. Regarding histogenesis of these type II tumors, the concept of resting/atrophic endometrial transformation into endometrial intraepithelial carcinoma has been proposed and increasing evidence has demonstrated that p53 features are associated with the dynamic transformation of endometrial carcinoma development (70). Serous carcinoma typically displays significant overexpression of p53 and p16 (71). There was a large difference in the functions of the DEGs between type I and type II endometrial cancer in the present study, which suggested that there were also great differences in the biological characteristics of these two types of endometrial cancer.

In conclusion, by comparing the type I and type II EC samples, a total of 9,671 DEGs, including 5,962 upregulated and 3,709 downregulated DEGs, were identified. The 13 top hub genes, MAPK1, PHLPP1, ESR1, MDM2, CDKN2A, CDKN1A, AURKA, BCL2L1, POLQ, PIK3R3, RHOQ, EIF4E and LATS2, were identified from the PPI network. The key pathways and genes associated with the histological types of EC, endometrioid adenocarcinoma and serous adenocarcinoma, were identified. Based on the enrichment analysis of DEGs, they were primarily associated with the positive 
regulation of apoptosis, programmed cell death and cell death. The altered DEGs (MAPK1, MDM2, AURKA, EIF4E and LATS2) may play roles in the tumor differentiation of EC. The present study highlights the novel molecular features of serous adenocarcinoma. These identified key genes may help to identify the potential molecular mechanism underlying tumorigenesis, and additionally serve as candidate biomarkers and potential targeted therapy key points for type II EC. However, the present study had a limitation, as the findings were not validated in clinical samples. More in-depth experimental studies are necessary to sufficiently confirm the role of these DEGs in these two different types of EC.

\section{Acknowledgements}

Not applicable.

\section{Funding}

The present study was funded by the Natural Science Foundation of China (grant nos. 81772790 and 81572568), the Postgraduate Innovation Fund of 13th Five-year comprehensive investment, Tianjin Medical University (grant no. YJSCX201812) and the Natural Science Foundation of Tianjin (grant no. 15JCYBJC25900).

\section{Availability of data and materials}

The datasets used and/or analyzed during the current study are available from the corresponding author on reasonable request.

\section{Authors' contributions}

$\mathrm{KZ}$ drafted this manuscript. $\mathrm{KZ}$ and $\mathrm{HL}$ conceived and designed the study. YY, KL and YZ collected the data and performed the analyses. YW and FX helped to design the study and revised the manuscript. All authors approved the final version of manuscript.

\section{Ethics approval and consent to participate}

Not applicable.

\section{Patient consent for publication}

Not applicable.

\section{Competing interests}

The authors declare that they have no competing interests.

\section{References}

1. Siegel RL, Miller KD and Jemal A: Cancer statistics, 2018. CA Cancer J Clin 68: 7-30, 2018.

2. Bokhman JV: Two pathogenetic types of endometrial carcinoma. Gynecol Oncol 15: 10-17, 1983.

3. Koh WJ, Abu-Rustum NR, Bean S, Bradley K, Campos SM, Cho KR, Chon HS, Chu C, Cohn D, Crispens MA, et al: Uterine neoplasms, version 1.2018, NCCN clinical practice guidelines in oncology. J Natl Compr Canc Netw 16: 170-199, 2018.
4. Buhtoiarova TN, Brenner CA and Singh M: Endometrial carcinoma: Role of current and emerging biomarkers in resolving persistent clinical dilemmas. Am J Clin Pathol 145: 8-21, 2016.

5. Felix AS, Yang HP, Bell DW and Sherman ME: Epidemiology of endometrial carcinoma: Etiologic importance of hormonal and metabolic influences. Adv Exp Med Biol 943: 3-46, 2017.

6. Mirakhor Samani S, Ezazi Bojnordi T, Zarghampour M, Merat S and Fouladi DF: Expression of p53, Bcl-2 and Bax in endometrial carcinoma, endometrial hyperplasia and normal endometrium: A histopathological study. J Obstet Gynaecol 38: 999-1004, 2018.

7. Chen HX, Xu XX, Tan BZ, Zhang Z and Zhou XD: MicroRNA-29b inhibits angiogenesis by targeting VEGFA through the MAPK/ERK and PI3K/Akt signaling pathways in endometrial carcinoma. Cell Physiol Biochem 41: 933-946, 2017.

8. Ashburner M, Ball CA, Blake JA, Botstein D, Butler $\mathrm{H}$, Cherry JM, Davis AP, Dolinski K, Dwight SS, Eppig JT, et al: Gene ontology: Tool for the unification of biology. The Gene Ontology Consortium. Nat Genet 25: 25-29, 2000.

9. Du J, Yuan Z, Ma Z, Song J, Xie X and Chen Y: KEGG-PATH: Kyoto encyclopedia of genes and genomes-based pathway analysis using a path analysis model. Mol Biosyst 10: 2441-2447, 2014.

10. Cancer Genome Atlas Research Network, Weinstein JN, Collisson EA, Mills GB, Shaw KR, Ozenberger BA, Ellrott K, Shmulevich I, Sander C and Stuart JM: The Cancer Genome Atlas pan-cancer analysis project. Nat Genet 45: 1113-1120, 2013.

11. Guan J, Xie L, Luo X, Yang B, Zhang H, Zhu Q and Chen X: The prognostic significance of estrogen and progesterone receptors in grade I and II endometrioid endometrial adenocarcinoma: Hormone receptors in risk stratification. J Gynecol Oncol 30: e13, 2019.

12. Tan W, Zhong Z, Carney RP, Men Y, Li J, Pan T and Wang Y: Deciphering the metabolic role of AMPK in cancer multi-drug resistance. Semin Cancer Biol 56: 56-71, 2019.

13. Nadaraja S, Jørgensen TL, Matzen LE and Herrstedt J: Impact of age, comorbidity, and FIGO stage on treatment choice and mortality in older danish patients with gynecological cancer: A retrospective register-based cohort study. Drugs Real World Outcomes 5: 225-235, 2018

14. Lax SF: New features in the 2014 WHO classification of uterine neoplasms. Pathologe 37: 500-511, 2016 (In German).

15. Kanehisa M, Goto S, Furumichi M, Tanabe $M$ and Hirakawa $M$ : KEGG for representation and analysis of molecular networks involving diseases and drugs. Nucleic Acids Res 38: D355-D360, 2010.

16. Szklarczyk D, Franceschini A, Wyder S, Forslund K, Heller D, Huerta-Cepas J, Simonovic M, Roth A, Santos A, Tsafou KP, et al: STRING v10: Protein-protein interaction networks, integrated over the tree of life. Nucleic Acids Res 43: D447-D452, 2015.

17. Ono K, Demchak B and Ideker T: Cytoscape tools for the web age: D3.js and Cytoscape.js exporters. F1000Res 3: 143, 2014.

18. Monterossi G, Ghezzi F, Vizza E, Zannoni GF, Uccella S, Corrado G, Restaino S, Quagliozzi L, Casarin J, Dinoi G, et al: Minimally invasive approach in type II endometrial cancer: Is it wise and safe? J Minim Invasive Gynecol 24: 438-445, 2017.

19. Liu Y, Nan F, Lu K, Wang Y, Liu Y, Wei S, Wu R and Wang Y: Identification of key genes in endometrioid endometrial adenocarcinoma via TCGA database. Cancer Biomark 21: 11-21, 2017.

20. Corbet C, Pinto A, Martherus R, Santiago DJJ, Polet F and Feron O: Acidosis drives the reprogramming of fatty acid metabolism in cancer cells through Changes in mitochondrial and histone acetylation. Cell Metab 24: 311-323, 2016.

21. Pan J, Cheng L, Bi X, Zhang X, Liu S, Bai X, Li F and Zhao AZ: Elevation of omega-3 polyunsaturated fatty acids attenuates PTEN-deficiency induced endometrial cancer development through regulation of COX-2 and PGE2 production. Sci Rep 5: $14958,2015$.

22. de Lorgeril M and Salen P: New insights into the health effects of dietary saturated and omega- 6 and omega- 3 polyunsaturated fatty acids. BMC Med 10: 50, 2012.

23. Zanoaga O, Jurj A, Raduly L, Cojocneanu-Petric R, FuentesMattei E, Wu O, Braicu C, Gherman CD and Berindan-Neagoe I: Implications of dietary omega- 3 and omega- 6 polyunsaturated fatty acids in breast cancer. Exp Ther Med 15: 1167-1176, 2018.

24. Edmondson RJ, Crosbie EJ, Nickkho-Amiry M, Kaufmann A, Stelloo E, Nijman HW, Leary A, Auguste A, Mileshkin L, Pollock P, et al: Markers of the p53 pathway further refine molecular profiling in high-risk endometrial cancer: A TransPORTEC initiative. Gynecol Oncol 146: 327-333, 2017. 
25. Nout RA, Bosse T, Creutzberg CL, Jürgenliemk-Schulz IM, Jobsen JJ, Lutgens LC, van der Steen-Banasik EM, van Eijk R, Ter Haar NT and Smit VT: Improved risk assessment of endometrial cancer by combined analysis of MSI, PI3K-AKT, Wnt/3-catenin and P53 pathway activation. Gynecol Oncol 126: 466-473, 2012

26. Theisen ER, Gajiwala S, Bearss J, Sorna V, Sharma S and Janat-Amsbury M: Reversible inhibition of lysine specific demethylase 1 is a novel anti-tumor strategy for poorly differentiated endometrial carcinoma. BMC Cancer 14: 752, 2014.

27. Gao X, Sun J, Huang C, Hu X, Jiang N and Lu C: RNAi-mediated silencing of NOX4 inhibited the invasion of gastric cancer cells through JAK2/STAT3 signaling. Am J Transl Res 9: 4440-4449, 2017.

28. Shen Y, Bian R, Li Y, Gao Y, Liu Y, Xu Y, Song X and Zhang Y: Liensinine induces gallbladder cancer apoptosis and G2/M arrest by inhibiting ZFX-induced PI3K/AKT pathway. Acta Biochim Biophys Sin (Shanghai): 607-614., 2019.

29. Lo RC, Leung CO, Chan KK, Ho DW, Wong CM, Lee TK and Ng IO: Cripto-1 contributes to stemness in hepatocellular carcinoma by stabilizing Dishevelled-3 and activating Wnt/beta-catenin pathway. Cell Death Differ 25: 1426-1441, 2018 .

30. Zhang J, Li Y, Dong M and Wu D: Long non-coding RNA NEAT1 regulates E2F3 expression by competitively binding to miR-377 in non-small cell lung cancer. Oncol Lett 14 4983-4988, 2017.

31. Yu T, Jia W, An Q, Cao X and Xiao G: Bioinformatic analysis of GLI1 and related signaling pathways in chemosensitivity of gastric cancer. Med Sci Monit 24: 1847-1855, 2018.

32. Zhang L, Feng T and Spicer LJ: The role of tight junction proteins in ovarian follicular development and ovarian cancer. Reproduction 155: R183-R198, 2018.

33. Martin TA, Watkins G, Mansel RE and Jiang WG: Loss of tight junction plaque molecules in breast cancer tissues is associated with a poor prognosis in patients with breast cancer. Eur J Cancer 40: 2717-2725, 2004.

34. Youakim A and Ahdieh M: Interferon-gamma decreases barrier function in T84 cells by reducing ZO-1 levels and disrupting apical actin. Am J Physiol 276: G1279-G1288, 1999.

35. Chen H, Wang Y and Xue F: Expression and the clinical significance of Wnt10a and Wnt10b in endometrial cancer are associated with the Wnt/ $\beta$-catenin pathway. Oncol Rep 29: 507-514, 2013

36. Han X, Cao Y, Wang $\mathrm{K}$ and Zhu G: HMGA1 facilitates tumor progression through regulating $\mathrm{Wnt} /$ beta-catenin pathway in endometrial cancer. Biomed Pharmacother 82: 312-318, 2016.

37. Wang ZM, Wan XH, Sang GY, Zhao JD, Zhu QY and Wang DM: miR-15a-5p suppresses endometrial cancer cell growth via Wnt/ $\beta$-catenin signaling pathway by inhibiting WNT3A. Eur Rev Med Pharmacol Sci 21: 4810-4818, 2017.

38. Wang T, Wang M, Fang S, Wang Q, Fang R and Chen J: Fibulin-4 is associated with prognosis of endometrial cancer patients and inhibits cancer cell invasion and metastasis via Wnt/ $\beta$-catenin signaling pathway. Oncotarget 8: 18991-19012, 2017.

39. Chen QG, Zhou W, Han T, Du SQ, Li ZH, Zhang Z, Shan GY and Kong CZ: MiR-378 suppresses prostate cancer cell growth through downregulation of MAPK1 in vitro and in vivo. Tumour Biol 37: 2095-2103, 2016.

40. Wei WT, Nian XX, Wang SY, Jiao HL, Wang YX, Xiao ZY, Yang RW, Ding YQ, Ye YP and Liao WT: miR-422a inhibits cell proliferation in colorectal cancer by targeting AKT1 and MAPK1. Cancer Cell Int 17: 91, 2017.

41. Wang Y, Gao C, Zhang Y, Gao J, Teng F, Tian W, Yang W, Yan Y and Xue F: Visfatin stimulates endometrial cancer cell proliferation via activation of PI3K/Akt and MAPK/ERK1/2 signalling pathways. Gynecol Oncol 143: 168-178, 2016

42. Kanan Y, Matsumoto H, Song H, Sokolov M, Anderson RE and Rajala RV: Serine/threonine kinase akt activation regulates the activity of retinal serine/threonine phosphatases, PHLPP and PHLPPL. J Neurochem 113: 477-488, 2010.

43. Li X, Stevens PD, Liu J, Yang H, Wang W, Wang C, Zeng Z, Schmidt MD, Yang M, Lee EY and Gao T: PHLPP is a negative regulator of RAF1, which reduces colorectal cancer cell motility and prevents tumor progression in mice. Gastroenterology 146 1301-1312.e1-e10, 2014

44. Huang YS, Chang CC, Lee SS, Jou YS and Shih HM: Xist reduction in breast cancer upregulates AKT phosphorylation via HDAC3-mediated repression of PHLPP1 expression. Oncotarget 7: 43256-43266, 2016.
45. Hribal ML, Mancuso E, Spiga R, Mannino GC, Fiorentino TV, Andreozzi F and Sesti G: PHLPP phosphatases as a therapeutic target in insulin resistance-related diseases. Expert Opin Ther Targets 20: 663-675, 2016.

46. Toderow V, Rahmeh M, Hofmann S, Kirn V, Mahner S, Jeschke U and von Schönfeldt V: Promotor analysis of ESR1 in endometrial cancer cell lines, endometrial and endometriotic tissue. Arch Gynecol Obstet 296: 269-276, 2017.

47. Jensen EV, Block GE, Smith S, Kyser K and DeSombre ER: Estrogen receptors and breast cancer response to adrenalectomy. Natl Cancer Inst Monogr 34: 55-70, 1971.

48. Zhang CZ, Wang SX, Zhang Y, Chen JP and Liang XM: In vitro estrogenic activities of Chinese medicinal plants traditionally used for the management of menopausal symptoms. J Ethnopharmacol 98: 295-300, 2005.

49. Hall KL, Teneriello MG, Taylor RR, Lemon S, Ebina M, Linnoila RI, Norris JH, Park RC and Birrer MJ: Analysis of Ki-ras, p53, and MDM2 genes in uterine leiomyomas and leiomyosarcomas. Gynecol Oncol 65: 330-335, 1997.

50. Jeczen R, Skomra D, Cybulski M, Schneider-Stock R, Szewczuk W, Roessner A, Rechberger T and Semczuk A: P53/MDM2 overexpression in metastatic endometrial cancer: Correlation with clinicopathological features and patient outcome. Clin Exp Metastasis 24: 503-511, 2007.

51. Wu JP and Luo X: The association between murine double minute 2 (MDM2) rs2279744 and endometrial cancer risk in a Chinese Han population. Cell Mol Biol (Noisy-le-grand) 63: 128-130, 2017.

52. Dutto I, Tillhon M, Cazzalini O, Stivala LA and Prosperi E: Biology of the cell cycle inhibitor p21(CDKN1A): Molecular mechanisms and relevance in chemical toxicology. Arch Toxicol 89: 155-178, 2015.

53. Zhao J, Shi L, Zeng S, Ma C, Xu W, Zhang Z, Liu Q, Zhang P, Sun Y and Xu C: Importin-11 overexpression promotes the migration, invasion, and progression of bladder cancer associated with the deregulation of CDKN1A and THBS1. Urol Oncol 36: 11.e1-311.e13, 2018.

54. Seleznik GM, Reding T, Peter L, Gupta A, Steiner SG, Sonda S, Verbeke CS, Dejardin E, Khatkov I, Segerer S, et al: Development of autoimmune pancreatitis is independent of CDKN1A/p21-mediated pancreatic inflammation. Gut 67: 1663-1673, 2018

55. Hayashi H, Kohno T, Ueno H, Hiraoka N, Kondo S, Saito M, Shimada Y, Ichikawa H, Kato M, Shibata T, et al: Utility of assessing the number of mutated KRAS, CDKN2A, TP53, and SMAD4 genes using a targeted deep sequencing assay as a prognostic biomarker for pancreatic cancer. Pancreas 46: 335-340, 2017.

56. Umene K, Banno K, Kisu I, Yanokura M, Nogami Y, Tsuji K, Masuda K, Ueki A, Kobayashi Y, Yamagami W, et al: Aurora kinase inhibitors: Potential molecular-targeted drugs for gynecologic malignant tumors. Biomed Rep 1: 335-340, 2013.

57. Tanaka Y, Ueda Y, Nakagawa S, Matsuzaki S, Kobayashi E, Shiki Y, Nishio Y, Takemura M, Yamamoto T, Sawada K, et al: A phase I/II study of GLIF combination chemotherapy for taxane/platinum-refractory/resistant endometrial cancer (GOGO-EM2). Cancer Chemother Pharmacol 82: 585-592, 2018.

58. Umene K, Yanokura M, Banno K, Irie H, Adachi M, Iida M, Nakamura K, Nogami Y, Masuda K, Kobayashi Y, et al: Aurora kinase A has a significant role as a therapeutic target and clinical biomarker in endometrial cancer. Int J Oncol 46: 1498-1506, 2015.

59. Inoue-Yamauchi A, Jeng PS, Kim K, Chen HC, Han S, Ganesan YT, Ishizawa K, Jebiwott S, Dong Y, Pietanza MC, et al: Targeting the differential addiction to anti-apoptotic BCL-2 family for cancer therapy. Nat Commun 8: 16078, 2017.

60. Lucas CM, Milani M, Butterworth M, Carmell N, Scott LJ, Clark RE, Cohen GM and Varadarajan S: High CIP2A levels correlate with an antiapoptotic phenotype that can be overcome by targeting BCL-XL in chronic myeloid leukemia. Leukemia 30 : 1273-1281, 2016

61. Sato K, Suzuki T, Yamaguchi Y, Kitade Y, Nagase T and Ueda H: PLEKHG2/FLJ00018, a Rho family-specific guanine nucleotide exchange factor, is tyrosine phosphorylated via the EphB2/cSrc signaling pathway. Cell Signal 26: 691-696, 2014.

62. Zhang L, Huang J, Yang N, Greshock J, Liang S, Hasegawa K, Giannakakis A, Poulos N, O'Brien-Jenkins A, Katsaros D, et al: Integrative genomic analysis of phosphatidylinositol 3'-kinase family identifies PIK3R3 as a potential therapeutic target in epithelial ovarian cancer. Clin Cancer Res 13: 5314-5321, 2007. 
63. Dong L and Hui L: HOTAIR promotes proliferation, migration, and invasion of ovarian cancer SKOV3 cells through regulating PIK3R3. Med Sci Monit 22: 325-331, 2016.

64. Zhang C, Min L, Liu J, Tian W, Han Y, Qu L and Shou C: Integrated analysis identified an intestinal-like and a diffuse-like gene sets that predict gastric cancer outcome. Tumour Biol: Nov 17, 2016 (Epub ahead of print).

65. Choi CH, Lee JS, Kim SR, Lee YY, Kim CJ, Lee JW, Kim TJ, Lee JH, Kim BG and Bae DS: Direct inhibition of eIF4E reduced cell growth in endometrial adenocarcinoma. J Cancer Res Clin Oncol 137: 463-469, 2011.

66. Shi ZM, Liu YN, Fu B, Shen YF and Li LM: Expression profile of eukaryotic translation initiation factor and matrix metalloproteinase 9 in endometrial cancer tissue. J Biol Regul Homeost Agents 31: 1053-1059, 2017.

67. Guo C, Liang C, Yang J, Hu H, Fan B and Liu X: LATS2 inhibits cell proliferation and metastasis through the Hippo signaling pathway in glioma. Oncol Rep 41: 2753-2761, 2019.

68. Zhao B, Li L, Wang L, Wang CY, Yu J and Guan KL: Cell detachment activates the Hippo pathway via cytoskeleton reorganization to induce anoikis. Genes Dev 26: 54-68, 2012.
69. Mitamura T, Watari H, Wang L, Kanno H, Kitagawa M, Hassan MK, Kimura T, Tanino M, Nishihara H, Tanaka S and Sakuragi N: microRNA 31 functions as an endometrial cancer oncogene by suppressing Hippo tumor suppressor pathway. Mol Cancer 13: 97, 2014.

70. Yasuda M: Immunohistochemical characterization of endometrial carcinomas: Endometrioid, serous and clear cell adenocarcinomas in association with genetic analysis. J Obstet Gynaecol Res 40: 2167-2176, 2014.

71. Alkushi A, Köbel M, Kalloger SE and Gilks CB: High-grade endometrial carcinoma: Serous and grade 3 endometrioid carcinomas have different immunophenotypes and outcomes. Int J Gynecol Pathol 29: 343-350, 2010.

(i) (9) This work is licensed under a Creative Commons Attribution-NonCommercial-NoDerivatives 4.0 International (CC BY-NC-ND 4.0) License. 